\title{
The efficacy and safety of compound glycyrrhizin combined with desloratadine in the treatment of chronic urticaria: protocol for a systematic review of randomized controlled trials
}

\section{Qiuyue Wang}

Hospital of Chengdu University of Traditional Chinese Medicine https://orcid.org/0000-0002-75004300

\section{Xingxin $\mathrm{Hu}$}

Hospital of Chengdu University of Traditional Chinese Medicine

Mao Li

Hospital of Chengdu University of Traditional Chinese Medicine

Qian Luo

Hospital of Chengdu University of Traditional Chinese Medicine

Pingsheng Hao ( $\nabla$ hpswl@126.com )

https://orcid.org/0000-0002-0376-6269

\section{Protocol}

Keywords: Compound glycyrrhizin, Desloratadine, Chronic urticaria, Systematic review, Meta-analysis

Posted Date: May 22nd, 2020

DOI: https://doi.org/10.21203/rs.3.rs-29774/v1

License: (c) (i) This work is licensed under a Creative Commons Attribution 4.0 International License.

Read Full License 


\section{Abstract}

Background: Chronic urticaria (CU) is a common skin disease characterized by a short-term (<24hours) spontaneous skin rash (urticaria) with or without angioedema that lasts longer than 6 weeks. CU is not life-threatening but has been shown to have a significant impact on the physical and mental health of patients. Because of chronic itching or physical discomfort in patients with $\mathrm{CU}$, symptoms such as the repeated occurrence of red, swollen, itchy, anxiety, insomnia, and psychological stress are prone to occur. Whereas, there is no related systematic review and meta-analysis. Thus, this systematic review protocol aims to describe a systematic review and meta-analysis to testify the efficacy and safety of compound glycyrrhizin (CG) combined with desloratadine in the treatment of CU.

Methods: Our systematic review will search all randomized controlled trials (RCTs) for CG combined with desloratadine in the treatment of $\mathrm{CU}$, electronically and manually, regardless of publication status and language, until January 14, 2020. Databases include PubMed, EMBASE, Web of Science, Cochrane Controlled Trials Register (CENTRAL), China National Knowledge Infrastructure (CNKI), China Biomedical Literature Database (CBM), Chinese Science Journal Database (VIP Database) and Wanfang database. Other sources, including reference lists of identified publications and meeting minutes, will also be searched. Manually search for grey literature, including unpublished conference articles. The main outcomes contain the total effective rate, the urticaria activity score (UAS), Itching score, the chronic urticaria quality of life questionnaire (CU-Q2oL), or other validated symptom scores and the effective rate and adverse events from baseline to the end of studies. This study will provide a comprehensive review of the available evidence for the treatment of $\mathrm{CU}$ with this therapy. We will assess the risk of bias with the Cochrane Risk of Bias Tool, narratively synthesize the extracted data and conduct a meta-analysis of studies with similar characteristics. Two independent raters will screen articles and assess the risk of bias.

Discussion: This study will provide a high-quality synthesis of the effects of compound glycyrrhizin (CG) combined with desloratadine in the treatment of $\mathrm{CU}$. We hope that this review will give more convincing proof to assist clinicians during the decision-making process when dealing with $\mathrm{CU}$.

\section{Systematic review registration: PROSPERO CRD42020165478}

\section{Background}

\section{Rationale}

Urticaria is a common clinical allergic disease, which is characterized by localized edema reaction caused by skin and mucosal small blood vessels dilatation and increased permeability. It is characterized by various wind groups accompanied by pruritus. About $20 \%$ of patients have angioedema.[1,2] Chronic urticaria (CU) was defined when the clinical course of the disease was $\geq 6$ weeks and the incidence frequency was $\geq 2$ times/week.[1]At present, with the continuous deterioration of the natural environment and the gradual occupation of unhealthy or sub-healthy lifestyle, the prevalence rate of CU is increasing 
year by year. According to statistics, the lifetime prevalence rate of urticaria is $7.8 \% \sim 22.3 \%$, and the time point prevalence rate is $0.5 \% \sim 1.0 \%$.[3] The etiology of $\mathrm{CU}$ is complex, and about $3 / 4$ of the patients could not find a clear etiology.[2] The pathogenesis of urticaria is complex and not clear at present, but it basically belongs to allergic disease, which is related to mast cells. Mast cells are the key effector cells in the pathogenesis of urticaria, which are induced by immune and non-immune mechanisms.[4,5] Mast cells activation and degranulation is the "central link" in the occurrence of this disease. Activated cells release three major metabolites: histamine, cytokines and chemokines, leukotrienes and prostaglandins, which affect the occurrence, development and prevention and treatment of urticaria. $\mathrm{CU}$ is also considered as a kind of psychological skin because of the serious physical and psychological impact of this disease on the patients.[6] The diagnosis can be made based on clinical manifestations, laboratory tests and pathological examinations. Western medicine treats patients education, etiology, treatment and symptom control are the main purposes to completely control urticaria symptoms and improve patients' quality of life.[1] The most commonly used drug is the second-generation $\mathrm{H} 1$ receptor antagonist, which can control the occurrence of $\mathrm{CU}$ during clinical use. Mauro Cataldi et al[7] considered in detail SecondGeneration H1-Antihistamines such as cetirizine, ebastine, fexofenadine, loratadine, desloratadine, mizolastine, and rupatadine and concluded that all these drugs have an excellent safety profile with no evidence of cardiotoxicity even when updated up to four times their standard licensed dose.[7] The main option in therapies aimed at symptomatic relief is to reduce the effect of mast cell mediators such as histamine, PAF, and others on the target organs. Many symptoms of urticaria are mediated primarily by the actions of histamine on $\mathrm{H} 1$-receptors located on endothelial cells and on sensory nerves. Thus, continuous treatment with $\mathrm{H} 1$-antihistamines is of eminent importance in the treatment of urticaria (safety data are available for use of several years continuously).[1]

Desloratadine, as $\mathrm{H} 1$ nonsedating antihistamines are first-line treatment options for $\mathrm{CU}$. The molecular formula of desloratadine is $\mathrm{C} 19 \mathrm{H} 19 \mathrm{CIN} 2$, which is the active metabolite of loratadine, which can effectively relieve the related diseases of CU. Desloratadine has a longer action time, stronger antihistamine effect, better anti-allergy effect, selective choline decomposability, difficulty in crossing the blood-brain barrier, no central sedative effect, and good patient compliance than other similar drugs.[8] For these reasons, it has been widely used as a first-line drug and a conventional antihistamine.

The roots and rhizomes of the perennial herb licorice (glycyrrhiza) are used in traditional medicine to treat a variety of diseases. In experimental and clinical studies, licorice has been shown to have a variety of pharmacological properties, including anti-inflammatory, antiviral, antibacterial, antioxidant, anti-diabetic, anti-asthmatic and anti-cancer activities, as well as immune regulation, protection of the stomach, the liver, the nerves, and the heart.[9-15] In recent years, some biochemical, molecular, and cellular mechanisms of licorice and its active components have also been confirmed by experimental studies.[16, 17] licorice is one of the key herbs used in Chinese medicine. Clinically, it has been used for a range of skin conditions including dermatitis, eczema, and psoriasis. $[18,19]$ CG is a manufactured product that contains glycyrrhizin and other ingredients (amino acetic acid, methionine), with glycyrrhizin (glycyrrhizic acid) being the key component. It is also known as Stronger Neo-Minophagen $\mathrm{C}$ and was originally used for improving liver function abnormalities in chronic liver disease in Japan.[20] Glycyrrhizin is the main 
component of the CG, which is metabolized into glycyrrhetinic acid in the body. It is similar to glucocorticoid in structure, but steroid has fewer adverse reactions.[21] Glycyrrhizin has been used as a traditional Chinese medicine for many centuries to treat allergic diseases.[22] Because of its antiviral and antiallergic properties, glycyrrhizin is a derivative of herbs used to treat hepatitis and allergic diseases.[23, 24] CG combined with desloratadine is widely used in the treatment of chronic urticaria in China. By inhibiting the inflammatory mediators released by desloratadine in chronic urticaria and regulating the immune mechanism of $C G$, we can jointly help patients to alleviate their condition and relieve the physiological pain brought by the disease.

\section{Objectives}

The purpose of this systematic review is to comprehensively collect high-quality randomized controlled trials (RCTs), analyze and summarize the evidence to evaluate the efficacy and safety of CG combined with desloratadine in the treatment of $\mathrm{CU}$. To provide evidence for clinical practice by evaluating the efficacy and safety through the Cochrane systematic evaluation. We may recommend an effective treatment that provides clinicians with clinical decision making options to help patients seek further treatment options.

\section{Methods}

This protocol is developed in accordance with the Preferred Reporting Items for Systematic Reviews and Meta-analyses Protocols (PRISMA-P) statement guidelines[25]. The PRISMA Extension Statement is used to ensure all aspects of methods and findings are reported.

\section{Eligibility criteria}

\section{Inc/usion criteria}

There will be no restrictions on the length of treatment and duration of follow-up. This systematic review will include high-quality RCTs in English or Chinese that evaluated the therapeutic effect and safety of CG combined with desloratadine in the treatment of CU. Without any date of dissemination or restriction of publication type. To RCTs, it should report adequate randomization methods, eligible diagnosis, eligible outcome measurement, and statistical methods description. Blinding will not be a part of the inclusion criteria because of the particularity of acupuncture manipulation.

\section{Exclusion criteria}

1) controlled (non-randomized) clinical trials, case reports, observational study, retrospective studies, animal mechanism studies, self-controlled, random crossover studies; 
2) Studies with overlapping data;

3) Studies missing raw data.

\section{Participants}

Regardless of gender, age, ethnicity, education, and economic status, patients with CU who meet the following diagnostic criteria (eg, EAACl/GA2LEN/ EDF/WAO guidelines, Chinese Medical Association clinical treatment guidelines. Skin disease and STD Volume, Practical Dermatology, Clinical dermatology.).[1]

\section{Intervention and comparators}

The intervention group was mainly treated with CG combined with desloratadine.

A comparison of the following processing will be performed:

1. CG combined with desloratadine compared with no treatment.

2. CG combined with desloratadine compared with placebo or sham drugs therapy.

3. CG combined with desloratadine compared with a separate desloratadine therapy.

4. CG combined with desloratadine compared with other active therapies.

\section{Outcomes}

Primary outcomes

The primary outcomes will be the total effective rate. According to the severity of clinical symptoms using 4 scores; the total score is the sum of the individual scores. Symptom Score Reducing Index (SSRI)=(total score before treatment-total score after treatment)/total score before treatment $\times 100 \%$.

Secondary outcomes

1. Itching score: The degree of itching was evaluated by Visual Analogue Scale (VAS).

2. The related inflammatory factors, such as interleukin-interferon, immunoglobulin $E(\operatorname{lgE})$, were observed

3. Quality of life, using the chronic urticaria quality of life questionnaire (CU-Q2oL).

4. Response rate.

5. Adverse events. Usually the last observation score change from baseline to follow-up. 


\section{Information sources}

A literature search strategy using medical subject headings and text words has been developed. We will search MEDLINE (OVID interface), EMBASE (OVID interface), and the Cochrane Central Register of Controlled Trials (Wiley interface).

To ensure the capture of all relevant trials, all selected studies will also undergo an ancestry search, in addition to citation search using SCOPUS. OpenGrey will be interrogated for unpublished relevant literature.

\section{Search strategy}

The systematic review will search all randomized controlled trials (RCTs) by searching the following database: PubMed, EMBASE, Web of Science, Cochrane Controlled Trials Register (CENTRAL), China National Knowledge Infrastructure (CNKI), China Biomedical Literature Database (CBM), Chinese Science Journal Database (VIP Database) and Wanfang database with a language limitation of English and Chinese until January 14, 2020. In addition, The related reference lists of identified publications, meeting minutes, gray literature, and unpublished literature for eligible studies which will be searched by us.

\section{Study selection}

All potential relevant clinical studies will be screened according to their titles, abstracts, keywords by 2 reviewers (QYW and $\mathrm{XXH}$ ) at the same time independently after removing duplicates and nonclinical trials. And then the intensive reading of full text could authenticate for further assessment if there are studies that could not be clearly included based on both titles and abstracts. Once any disagreement occurs, a decision will be resolved through discussion among the 2 reviewers (QYW and XXH), or argument will be adjudicated by a third reviewer (PSH). Details of entire study selection procedure are summarized in the flow chart (Fig. 1).

\section{Data extraction and management}

Before data extraction, a standard data extraction form containing specified outcomes will be created according to the inclusion. Two reviewers (QYW and $\mathrm{ML}$ ) will extract data independently from each trial: article general information, participants' characteristics (such as age, sex, race, disease history), number of participants on each group, intervention measure of trial and control group, outcomes measures. Any disagreements will be resolved through discussion or consultation between the 2 reviewers if necessary, final determination from a third reviewer (PSH) will be sought. When certain dates don't be provided in the paper, we will contact the original author for the needed information. 


\section{Risk of bias (quality) assessment}

Two reviewers (QYW and ML) will evaluate the risk of bias according to the risk of bias (ROB) tool[26] to assess the bias risk of all included studies. We will assess the risk of bias in the following areas: random sequence generation, concealment of allocation sequences, the blindness of participants and staff and their result evaluators, incomplete outcome data, selective outcome reports, and other sources of bias. This review will use $L, U$, and $H$ as the key to these assessments, where $L$ (low) indicates a lower risk of bias, $\mathrm{U}$ (unclear) indicates an uncertain risk of bias, and $\mathrm{H}$ (high) indicates a higher risk of bias. All reviewers will resolve their differences through discussions. The information contained in the study on the risk of biased assessments will be summarized in a tabular format with a critical discussion of results and impacts. If the information is unclear, we will try to contact the author. For republished articles, we only select the original text.

\section{Strategy for data synthesis}

Data analysis and quantitative data synthesis will be performed using RevMan V.5.3. For continuous data, if there is no heterogeneity, we will use mean difference (MD) or standard MD (SMD) to measure the therapeutic effect of $95 \%$ Cls. If significant heterogeneity is found, a random-effects model will be used. For the two-category data, we will use the $95 \%$ Cls hazard ratio (RR) for analysis. We will include data from parallel-group design studies for meta-analysis. Only the first phase of the data will be included in the random crossover trial. In these trials, participants were randomly divided into two intervention groups and individual measurements for each outcome of each participant were collected and analyzed. The result will be expressed as the RR of the binary data and the SMD of the continuous data.

\section{Dealing with missing data or unclear information.}

Referring to the Cochrane handbook for systematic reviews of intervention, if there are insufficient details or missing data in relation to the characteristics of the studies, 2 reviewers (QYW and XXH) will attempt to contact both senior and/or corresponding author of articles through email or telephone for further information about any missing data or unclear information. If it is not possible to contact the original authors or obtain sufficient information, we will exclude such studies and only analyze the available data and describe it in the discussion. The potential impact of insufficient data on the review results will be taken into account in the discussion section.

\section{Data synthesis and assessment for heterogeneity}

We will use RevMan V.5.3. for all statistical analyses. The forest map is used to illustrate the relative strength of the effect. The funnel plot is used to illustrate the bias because the number of trials exceeds 10. If a significant difference is detected, a random-effects model will be used. If the $l^{2}$ test is less than 
$50 \%$, a fixed-effect model is used for data synthesis. If the $\mathrm{I}^{2}$ test is between $50 \%$ and $75 \%$, a randomeffects model is used for data synthesis. If the $\mathrm{I}^{2}$ test is higher than $75 \%$, we will investigate the possible causes from a clinical and methodological perspective and provide a descriptive analysis or a subgroup analysis.

When there are enough studies, we will conduct a sensitivity analysis of the main results according to the sample size, heterogeneous quality and statistical model (random or fixed effect model) to explore the robustness of the conclusions. Sensitivity analysis will be conducted by removing low-quality studies. If heterogeneity still exists after subgroup analysis, a meta-analysis will be performed again after excluding low-quality tests according to the STRICTA checklist. The results of these meta-analyses will be compared and discussed in terms of their sample size, the strength of the evidence, and their impact on the size of the merger effect. However, if there is a high risk of bias in all included studies, we will not conduct sensitivity analysis.

In terms of grading the quality of evidence, we will assess the evidence quality and credibility of the major findings (major outcomes and adverse events) of the studies included in our review, based on recommendations, assessments, development and grading of assessment (grade) guidelines[26]. The quality of evidence will be classified as "very low", "low", "medium" or "high". Any differences will be resolved by consensus or with the third review author ( $\mathrm{PSH})$.

\section{Patient and public involvement}

This systematic review and meta-analysis will be based on published studies; therefore, primary patient data will not be collected. Patients and the public will not be involved in the study design, recruitment, and data analysis.

\section{Ethics and dissemination}

The systematic review and meta-analysis don't require to pass the ethics approval. Because we include published articles rather than directly adopt interventions in participants. Ultimately, we will publish the results at a peer-reviewed journal follow as our study is completed. 
Table 1

Search strategy used in PubMed

\begin{tabular}{|c|c|}
\hline No & Search items \\
\hline 1 & Randomised controlled trial. pt \\
\hline 2 & Controlled clinical trial. pt \\
\hline 3 & Randomised. ti, ab \\
\hline 4 & Randomly. ti, ab \\
\hline 5 & Placebo. ti, ab \\
\hline 6 & Trial. ti, ab \\
\hline 7 & Groups. ti, ab \\
\hline 8 & 1 or $2-7$ \\
\hline 9 & Compound, ti. ab \\
\hline 10 & Glycyrrhizin. Mesh \\
\hline 11 & Glycyrrhizic Acid, ti. ab \\
\hline 12 & Acid, Glycyrrhizic, ti. ab \\
\hline 13 & Glycyrrhizinic Acid, ti. ab \\
\hline 14 & Acid, Glycyrrhizinic, ti. ab \\
\hline 15 & Glycyrrhizin, ti. ab \\
\hline 16 & Zinc Glycyrrhizate, ti. ab \\
\hline 17 & Glycyrrhizate, Zinc, ti. ab \\
\hline 18 & Diammonium Glycyrrhizinate, ti. ab \\
\hline 19 & Glycyrrhizinate, Diammonium, ti. ab \\
\hline 20 & Dipotassium Glycyrrhizinate, ti. ab \\
\hline 21 & Glycyrrhizinate, Dipotassium, ti. ab \\
\hline 22 & 10 or $11-21$ \\
\hline 23 & Desloratadine. Mesh \\
\hline 24 & descarboethoxyloratadine, ti. ab \\
\hline 25 & SCH-34117, ti. ab \\
\hline 26 & SCH 34117, ti. ab \\
\hline 27 & Clarinex, ti. ab \\
\hline
\end{tabular}




\begin{tabular}{|cl|}
\hline 28 & Neoclarityn, ti. ab \\
\hline 29 & Aerius, ti. ab \\
\hline 30 & 23 or $24-29$ \\
\hline 31 & chronic urticaria. Mesh \\
\hline 32 & Urticaria, Chronic, ti. ab \\
\hline 33 & Chronic Urticarias, ti. ab \\
\hline 34 & Chronic Hives, ti. ab \\
\hline 35 & Hives, ti. ab \\
\hline 36 & 31 or $32-35$ \\
\hline 37 & 8 and 9 and 22 and 30 and 36 \\
\hline
\end{tabular}

\section{Discussion}

CU has a high incidence, its course of the disease is long, recurrent, seriously affect the daily life of patients. This kind of disease happens time suddenly, disappears now and then, after subsiding, has no trace. Severe itching can lead to repeated scratching, which can lead to further infection and deterioration. The pathogenesis of $\mathrm{CU}$ is not clear, the etiology is complex, resulting in the treatment is very difficult, the treatment process is long.

The treatment of CU preferred antihistamine drugs, desloratadine as a new type of antihistamine drugs, its mechanism of action of drugs is through the conversion of desloratadine, making it blocking receptors with high selectivity, to suppress the body of the release of inflammatory mediators, the expression of adhesion factor, thus reducing patients, bacteria, such as excessive reaction to drugs. It has high safety, will not cause serious adverse reactions in patients, little impact on patients' daily life. In addition, glycyrrhizin contained in compound glycyrrhizin tablets inhibits the production of leukotrienes, prostaglandins, and other inflammatory mediums through selective enzymes and metabolic enzymes, helping patients to regulate immune function and fight inflammation and allergy.

However, the evidence for the combination of the two drugs is lacking, and a systematic evaluation will be conducted for the first time. We hope that this review will give more convincing proof to assist clinicians during the decision-making process when dealing with $\mathrm{CU}$.

\section{Abbreviations}

CU: Chronic urticaria; CG:Compound glycyrrhizin; RCTs: Randomized controlled trials; CENTRAL: Cochrane Controlled Trials Register; CNKI: China National Knowledge Infrastructure; CBM: China 
Biomedical Literature Database; VIP: Chinese Science Journal Database; UAS: The urticaria activity score; CU-Q2oL: The chronic urticaria quality of life questionnaire

\section{Declarations}

\section{Acknowledgements}

Not applicable.

\section{Authors' contributions}

The study was conceived by QYW and PSH. XXH, ML, and QL developed the eligibility criteria, search strategy, assessment of methodological quality, data extraction methods and data summary plan. QYW and XXH wrote the protocol. PSH supervised the work. All authors approved the final version.

\section{Funding}

This study was supported by the Scientific Research Foundation of Hospital of Chengdu University of Traditional Chinese Medicine.

\section{Availability of data and materials}

Not applicable.

\section{Ethics approval and consent to participate}

No ethics approval is required for this systematic review and meta-analysis because we will be using information from published studies. Our findings will be published in a peer-reviewed journal according to the PRISMA guidelines.

\section{Consent for publication}

Not applicable.

\section{Competing interests}

The authors declare that they have no competing interests. 


\section{References}

1. Zuberbier T, Aberer W, Asero R, Abdul Latiff AH, Baker D, Ballmer-Weber B, Bernstein JA, BindslevJensen C, Brzoza Z, Buense Bedrikow R et al: The EAACl/GA²LEN/EDF/WAO guideline for the definition, classification, diagnosis and management of urticaria. Allergy 2018, 73(7):1393-1414.

2. Hon KL, Leung AKC, Ng WGG, Loo SK: Chronic Urticaria: An Overview of Treatment and Recent Patents. Recent Pat Inflamm Allergy Drug Discov 2019, 13(1):27-37.

3. Godse K, De A, Zawar V, Shah B, Girdhar M, Rajagopalan M, Krupashankar DS: Consensus Statement for the Diagnosis and Treatment of Urticaria: A 2017 Update. Indian J Dermatol 2018, 63(1):2-15.

4. Pałgan K, Bartuzi Z: Platelet activating factor in allergies. Int J Immunopathol Pharmacol 2015, 28(4):584-589.

5. Wang D, Tang H, Shen Y, Wang F, Lin J, Xu J: Activation of the Blood Coagulation System in Patients with Chronic Spontaneous Urticaria. Clin Lab 2015, 61(9):1283-1288.

6. Ozkan M, Oflaz SB, Kocaman N, Ozseker F, Gelincik A, Büyüköztürk S, Ozkan S, Colakoğlu B: Psychiatric morbidity and quality of life in patients with chronic idiopathic urticaria. Ann Allergy Asthma Immunol 2007, 99(1):29-33.

7. Cataldi M, Maurer M, Taglialatela M, Church MK: Cardiac safety of second-generation H(1) antihistamines when updosed in chronic spontaneous urticaria. Clin Exp Allergy 2019, 49(12):16151623.

8. Geha RS, Meltzer EO: Desloratadine: A new, nonsedating, oral antihistamine. J Allergy Clin Immunol 2001, 107(4):751-762.

9. Li H, Guo D, Zhang L, Feng X: Glycyrrhizin attenuates histamine-mediated MUC5AC upregulation, inflammatory cytokine production, and aquaporin 5 downregulation through suppressing the NF-KB pathway in human nasal epithelial cells. Chem Biol Interact 2018, 285:21-26.

10. Yu Z, Wu F, Tian J, Guo X, An R: Protective effects of compound ammonium glycyrrhizin, L-arginine, silymarin and glucurolactone against liver damage induced by ochratoxin A in primary chicken hepatocytes. Mol Med Rep 2018, 18(3):2551-2560.

11. Li W, Li Y, Jiang X, Li X, Yu Z: Compound Ammonium Glycyrrhizin Protects Hepatocytes from Injury Induced by Lipopolysaccharide/Florfenicol through a Mitochondrial Pathway. Molecules 2018, 23(9).

12. Yu Z, Ohtaki Y, Kai K, Sasano T, Shimauchi H, Yokochi T, Takada H, Sugawara S, Kumagai K, Endo Y: Critical roles of platelets in lipopolysaccharide-induced lethality: effects of glycyrrhizin and possible strategy for acute respiratory distress syndrome. Int Immunopharmacol 2005, 5(3):571-580.

13. Shah SL, Wahid F, Khan N, Farooq U, Shah AJ, Tareen S, Ahmad F, Khan T: Inhibitory Effects of Glycyrrhiza glabra and Its Major Constituent Glycyrrhizin on Inflammation-Associated Corneal Neovascularization. Evid Based Complement Alternat Med 2018, 2018:8438101.

14. Qiao X, Wang Q, Wang S, Kuang Y, Li K, Song W, Ye M: A 42-Markers Pharmacokinetic Study Reveals Interactions of Berberine and Glycyrrhizic Acid in the Anti-diabetic Chinese Medicine Formula GegenQinlian Decoction. Front Pharmacol 2018, 9:622. 
15. Su XQ, Wang XY, Gong FT, Feng M, Bai JJ, Zhang RR, Dang XQ: Oral treatment with glycyrrhizin inhibits NLRP3 inflammasome activation and promotes microglial M2 polarization after traumatic spinal cord injury. Brain Res Bull 2020, 158:1-8.

16. Hosseinzadeh H, Nassiri-AsI M: Pharmacological Effects of Glycyrrhiza spp. and Its Bioactive Constituents: Update and Review. Phytother Res 2015, 29(12):1868-1886.

17. Pastorino G, Cornara L, Soares S, Rodrigues F, Oliveira M: Liquorice (Glycyrrhiza glabra): A phytochemical and pharmacological review. Phytother Res 2018, 32(12):2323-2339.

18. Saeedi M, Morteza-Semnani K, Ghoreishi MR: The treatment of atopic dermatitis with licorice gel. J Dermatolog Treat 2003, 14(3):153-157.

19. Morteza-Semnani K, Saeedi M, Shahnavaz B: Comparison of antioxidant activity of extract from roots of licorice (Glycyrrhiza glabra L.) to commercial antioxidants in $2 \%$ hydroquinone cream. $J$ Cosmet Sci 2003, 54(6):551-558.

20. Abe K, Ikeda T, Wake K, Sato T, Sato T, Inoue H: Glycyrrhizin prevents of lipopolysaccharide/Dgalactosamine-induced liver injury through down-regulation of matrix metalloproteinase-9 in mice. $J$ Pharm Pharmacol 2008, 60(1):91-97.

21. Asl MN, Hosseinzadeh H: Review of pharmacological effects of Glycyrrhiza sp. and its bioactive compounds. Phytother Res 2008, 22(6):709-724.

22. Xu W, Li Y, Ju M, Lai W, Lu X, Shi H, Shi W, Gu H, Li L: A Multicenter, Randomized, Double-Blind, Placebo-Controlled Study of Compound Glycyrrhizin Capsules Combined with a Topical Corticosteroid in Adults with Chronic Eczema. Evid Based Complement Alternat Med 2020, 2020:6127327.

23. Mou K, Pan W, Han D, Wen X, Cao F, Miao Y, Li P: Glycyrrhizin protects human melanocytes from H2O2-induced oxidative damage via the Nrf2-dependent induction of HO-1. Int J Mol Med 2019, 44(1):253-261.

24. Han S, Sun L, He F, Che H: Anti-allergic activity of glycyrrhizic acid on IgE-mediated allergic reaction by regulation of allergy-related immune cells. Sci Rep 2017, 7(1):7222.

25. Moher D, Shamseer L, Clarke M, Ghersi D, Liberati A, Petticrew M, Shekelle P, Stewart LA: Preferred reporting items for systematic review and meta-analysis protocols (PRISMA-P) 2015 statement. Syst Rev 2015, 4:1.

26. Cumpston M, Li T, Page MJ, Chandler J, Welch VA, Higgins JP, Thomas J: Updated guidance for trusted systematic reviews: a new edition of the Cochrane Handbook for Systematic Reviews of Interventions. Cochrane Database Syst Rev 2019, 10:Ed000142.

\section{Figures}




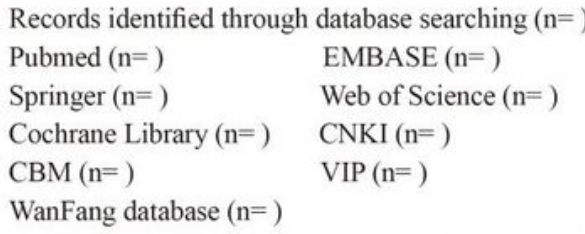

WanFang database $(\mathrm{n}=)$

Records identified through

other sources $(\mathrm{n}=)$

Identified publications $(\mathrm{n}=$ )

Meeting minutes $(\mathrm{n}=)$

Grey literature $(n=)$

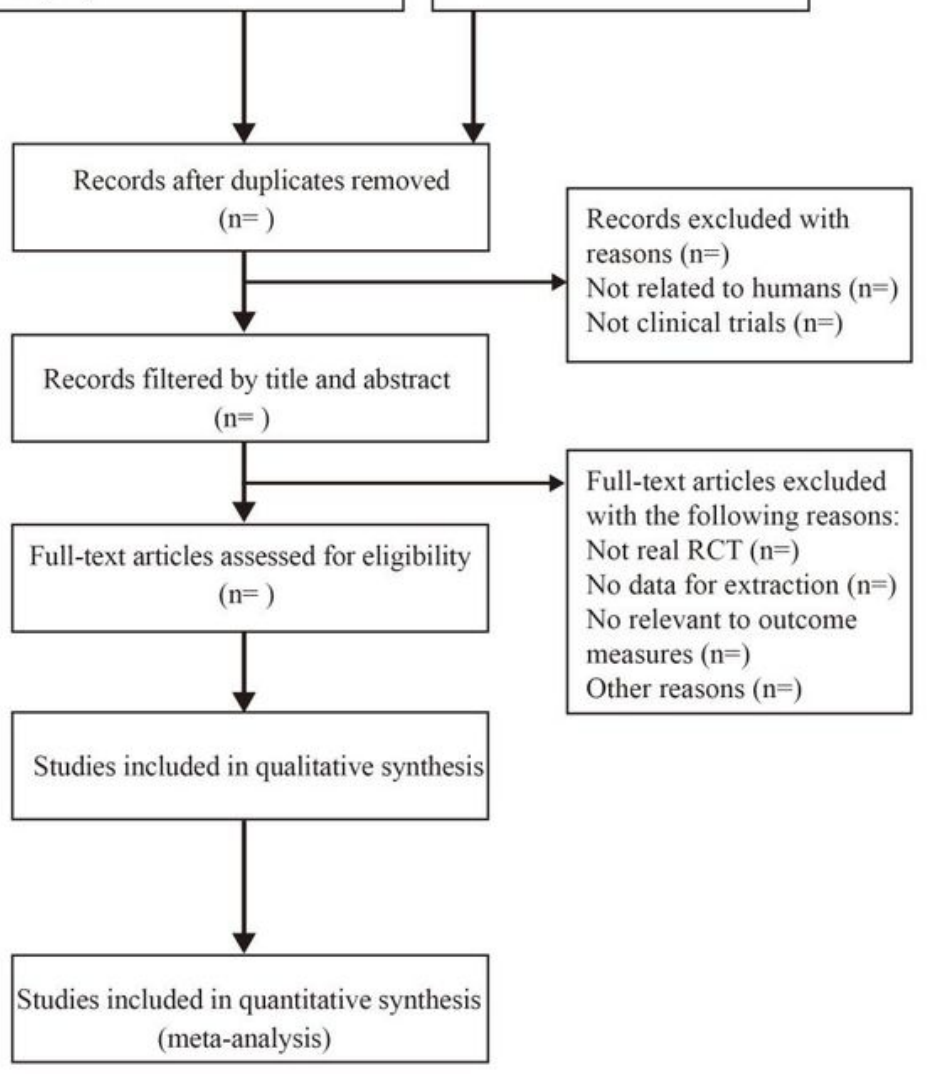

\section{Figure 1}

Flow diagram of studies identified

\section{Supplementary Files}

This is a list of supplementary files associated with this preprint. Click to download. 
- PRISMAPchecklist.doc

- PersonalCoverPingshengHao.docx 